\title{
Stokes First Problem for an Unsteady MHD Third-Grade Fluid in a Non-Porous Half Space with Hall Currents
}

\author{
Haider Zaman, Arif Sohail, Ubaidullah \\ Faculty of Numerical Sciences, Islamia College University, Peshawar, Pakistan \\ Email: haiderzaman67@yahoo.com
}

Received 6 January 2014; revised 11 February 2014; accepted 20 February 2014

Copyright (C) 2014 by authors and Scientific Research Publishing Inc.

This work is licensed under the Creative Commons Attribution International License (CC BY). http://creativecommons.org/licenses/by/4.0/

(c) (i) Open Access

\begin{abstract}
The well-known problem of unidirectional plane flow of a fluid in a non-porous half-space due to the impulsive motion of the rigid plane wall it rests upon is discussed in the context of an unsteady MHD third-grade fluid in presence of Hall currents. The governing non-linear partial differential equations describing the problem are converted to a system of non-linear ordinary differential equations by using the similarity transformations. The complex analytical solution is found by using the homotopy analysis method (HAM). The existing literature on the topic shows that it is the first study regarding the effects of Hall current on flow of an unsteady MHD third-grade fluid over an impulsively moving plane wall. The convergence of the obtained complex series solutions is carefully analyzed. The effects of dimensionless parameters on the velocity are illustrated through plots and the effects of the pertinent parameters on the local skin friction coefficient at the surface of the wall are presented numerically in tabular form.
\end{abstract}

\section{Keywords}

Stokes First Problem; Hall Currents; Unsteady; Third-Grade Fluid; HAM

\section{Introduction}

Almost every student of fluid mechanics is familiar with Stokes' first problem [1], the flows over a plane wall which is initially at rest and is suddenly set into motion in its own plane with a constant velocity is termed as Stokes' first (or Rayleigh-type) problem [2] [3]. Teipel [4] discussed impulsive motion of a flat plate in a viscoelastic fluid. Puri [5] investigated impulsive motion of a flat plate in a Rivlin-Ericksen fluid. Erdogan [6] analyzed plane surface suddenly set in motion in a non-Newtonian Fluid. Zeng and Weinbaum [7] investigated 
Stokes problems for moving half-planes. Tan and Xu [8] [9] investigated Stokes' first problem not only for generalized second grade fluid but also for generalized Maxwell fluid. Erdogan [10] analyzed the unsteady unidirectional flows generated by impulsive motion of a boundary or sudden application of a pressure gradient. Fetecau and Fetecau [11] solved Stokes' first problem for ordinary Oldroyd-B fluid by sine transform. Stokes' first problem for Oldroyd-B and second grade fluid in a porous half space is studied by Tan and Masuoka [12] [13]. Zierep and Fetecau [14] gave energetic balance for the Rayleigh-Stokes problem of a second grade fluid. Zierep et al. [15] discussed Rayleigh-Stokes problem for non-Newtonian medium with memory. Vieru et al. [16] found exact solution corresponding to the first problem of Stokes for Oldroyd-B fluid. Stokes' first problem for the rotating flow of a third grade fluid is numerically solved by Shahzad et al. [17]. Hayat et al. [18] presented numerical solution of Stokes' first problem for a third grade fluid in a porous half space. Fakhari et al. [19] presented a note on the interplay between symmetries, reduction and conservation laws of Stokes' first problem for third-grade rotating fluids. Sajid et al. [20] discussed Stokes' first problem for a MHD third grade fluid in a porous half space. The theoretical study of the effects of Hall current on flow of non-Newtonian fluids has been a subject of great interest to researchers because of its various applications in power generators and pumps, Hall accelerators, refrigeration coils, electric transformers, in flight MHD, solar physics involved in the sunspot development, the solar cycle, the structure of magnetic stars, electronic system cooling, cool combustors, fiber and granular insulation, oil extraction, thermal energy storage and flow through filtering devices and porous material regenerative heat exchangers. In presence of a strong magnetic field in an ionized gas of low density, the conductivity normal to the magnetic field is decreased by free spiraling of electrons and ions about the magnetic lines of force before suffering collisions. The phenomenon, well known in the literature, is called the Hall effect and a current induced in a direction normal to the electric and magnetic fields is called Hall current [21]. The Hall term representing the Hall current was ignored most of the time while applying Ohm's law, because it has no markable effect for small and moderate values of the magnetic field. The effects of Hall current are very important if the strong magnetic field is applied [22], because for strong magnetic field electromagnetic force is noticeable. The recent investigation for the applications of MHD is towards a strong magnetic field, due to which study of Hall current is important [23]-[36].

The present investigation is to analyze the Stokes first problem for an unsteady MHD third-grade fluid in a non-porous half space with Hall currents. The arising non-linear problem is solved by the homotopy analysis method (HAM), which is a novel technique and has been used by many researchers [37]-[45]. The method gives more realistic complex analytic solution which is uniformly valid for all values of the dimensionless time. The convergence region for the complex series solution is found with the help of $\hbar$-curve. The effects of the material parameters of the third-grade fluid, Hall parameter, Hartmann number and homotopy parameter on the velocity and its time series are investigated for the impulsive motion of the wall. When we introduce Hall currents in the problem then velocity field becomes complex and it has two parts, real and imaginary. It is not possible to plot complex velocity as a whole on the real plane, but to analyze the flow we plot real and imaginary parts separately. Sometimes our graphs for real and imaginary parts of velocity look likes that they are not satisfying boundary conditions, but it is not true because we are not plotting the complete solution, we are taking either real part or imaginary part of the solution.

\section{Mathematical Formulation of the Problem}

We consider the unsteady flow of an electrically conducting incompressible third-grade fluid past a rigid plane wall coinciding with the plane $y=0$. The fluid over the plane wall is initially at rest and it sets in motion due to the sudden jerked of the wall subjected to a uniform transverse magnetic field. It is assumed that the flow takes place in the upper half plane $y>0$ with the wall on the $x$-axis. The Cauchy stress tensor $\boldsymbol{T}$ for a third-grade fluid is given as [46]

$$
\boldsymbol{T}=-p \boldsymbol{I}+\mu \boldsymbol{A}_{1}+\alpha_{1} \boldsymbol{A}_{2}+\alpha_{2} \boldsymbol{A}_{1}^{2}+\beta_{1} \boldsymbol{A}_{3}+\beta_{2}\left(\boldsymbol{A}_{1} \boldsymbol{A}_{2}+\boldsymbol{A}_{2} \boldsymbol{A}_{1}\right)+\beta_{3}\left(\operatorname{tr} \boldsymbol{A}_{1}^{2}\right) \boldsymbol{A}_{1},
$$

where $p$ is the scalar pressure, $\boldsymbol{I}$ is the identity tensor, $\mu$ is the coefficient of viscosity, $\alpha_{i}, \beta_{j}$ $(i=1,2$ and $j=1,2,3)$ are the material parameters of third-grade fluid, and $\boldsymbol{A}_{i}(i=1,2,3)$ are the first three Rivlin-Ericksen tensors defined by [46]

$$
\boldsymbol{A}_{1}=(\operatorname{grad} \boldsymbol{V})+(\operatorname{grad} \boldsymbol{V})^{\mathrm{T}}
$$




$$
\boldsymbol{A}_{n+1}=\frac{\mathrm{d} \boldsymbol{A}_{n}}{\mathrm{~d} t}+\boldsymbol{A}_{n}(\operatorname{grad} \boldsymbol{V})+(\operatorname{grad} \boldsymbol{V})^{\mathrm{T}} \boldsymbol{A}_{n}, n=1,2 .
$$

The equations governing the magnetohydrodynamic flow with Hall effect are:

Velocity field:

$$
\boldsymbol{V}=[u(y, t), 0,0],
$$

Continuity equation:

$$
\operatorname{div} \boldsymbol{V}=0,
$$

Equation of motion:

$$
\rho \frac{\mathrm{d} \boldsymbol{V}}{\mathrm{d} t}=\operatorname{div}(\boldsymbol{T})+(\boldsymbol{J} \times \boldsymbol{B}),
$$

Maxwell equations:

$$
\operatorname{div} \boldsymbol{B}=0, \operatorname{Curl} \boldsymbol{B}=\mu_{m} \boldsymbol{J}, \operatorname{Curl} \boldsymbol{E}=-\frac{\partial \boldsymbol{B}}{\partial t}
$$

Generalized Ohm’s law:

$$
\boldsymbol{J}+\frac{w_{e} \tau_{e}}{\boldsymbol{B}_{0}} \boldsymbol{J} \times \boldsymbol{B}=\sigma(\boldsymbol{E}+\boldsymbol{V} \times \boldsymbol{B}),
$$

where $u(y, t)$ is the velocity component in the $x$-direction, $t$ is time, $\boldsymbol{B}\left(=\boldsymbol{B}_{0}+\boldsymbol{b}\right)$ is the total magnetic field, $\boldsymbol{B}_{0}$ is the applied magnetic field, $\boldsymbol{b}$ is the induced magnetic field, $\boldsymbol{J}$ is the current density, $\sigma$ is the electrical conductivity of the fluid, $\boldsymbol{E}$ is the electric field, $\mu_{m}$ is the magnetic permeability, $\rho$ is the fluid density, $w_{e}$ and $\tau_{e}$ are the cyclotron frequency and collision time of the electrons respectively. We assume that, the quantities $\rho, \mu_{m}$ and $\sigma$ are constants throughout the flow field, the magnetic field $\boldsymbol{B}$ is normal to the velocity vector $V$ and the induced magnetic field is neglected compared with the imposed magnetic field, so that the magnetic Reynolds number is small [47]. We also suppose that $w_{e} \tau_{e} \approx O(1)$ and $w_{i} \tau_{i} \ll 1$ (where $w_{i}$ and $\tau_{i}$ are the cyclotron frequency and collision time for ions respectively). The velocity is zero far from the wall and the pressure is uniform, so we neglect the pressure gradient term as the flow is the only kept in motion by the motion of the wall. Continuity Equation (5) is identically satisfied by the velocity taken in Equation (4). Under the aforementioned assumptions the unsteady Stokes first problem with Hall currents become

$$
\frac{\partial u}{\partial t}=v \frac{\partial^{2} u}{\partial y^{2}}-\frac{\sigma B_{0}^{2}(1+i \in)}{\rho\left(1+\epsilon^{2}\right)} u+\frac{\alpha_{1}}{\rho} \frac{\partial^{3} u}{\partial y^{2} \partial t}+\frac{\beta_{1}}{\rho} \frac{\partial^{4} u}{\partial y^{2} \partial t^{2}}+\frac{6\left(\beta_{2}+\beta_{3}\right)}{\rho}\left(\frac{\partial u}{\partial y}\right)^{2} \frac{\partial^{2} u}{\partial y^{2}},
$$

The boundary and initial conditions are

$$
\begin{aligned}
& u(y, t)=u_{w}=U, \quad \text { at } y=0, \text { for } t>0, \\
& u(y, t) \rightarrow 0 \text { as } y \rightarrow \infty, \\
& u(y, t)=0, \text { at } t=0, \text { for } y>0
\end{aligned}
$$

where $v$ is the kinematic viscosity, $\in\left(=w_{e} \tau_{e}\right)$ is the Hall parameter and $U$ is the velocity of the wall. In order to non-dimensionalize the problem let us introduce the similarity transformations

$$
u=U f(\eta, \xi), \eta=\frac{U}{v} y, \xi=\frac{U^{2}}{v} t,
$$

where $f(\eta, \xi)$ is the dimensionless velocity function, $\eta$ is the dimensionless distance from the wall and $\xi$ is the dimensionless time. Equations (9)-(11) become

$$
f^{\prime \prime}+\alpha \frac{\partial f^{\prime \prime}}{\partial \xi}+\beta \frac{\partial^{2} f^{\prime \prime}}{\partial \xi^{2}}-\frac{N(1+i \in)}{\left(1+\epsilon^{2}\right)} f+\zeta f^{\prime 2} f^{\prime \prime}-\frac{\partial f}{\partial \xi}=0
$$




$$
\begin{aligned}
& f(0, \xi)=1, f(\infty, \xi)=0, \text { for } \xi>0, \\
& f(\eta, 0)=0, \text { for } \eta>0
\end{aligned}
$$

where prime denotes differentiation with respect to $\eta, \alpha\left(=\alpha_{1} U^{2} / \rho v^{2}\right)$ is dimensionless second-grade parameter, $\beta\left(=\beta_{1} U^{4} / \rho v^{3}\right), \zeta\left(=6\left(\beta_{2}+\beta_{3}\right) U^{4} / \rho v^{3}\right)$ are dimensionless third-grade fluid material parameters and $N\left(=\sigma B_{0}^{2} v / \rho U^{2}\right)$ is the dimensionless modified Hartmann number [47]. The local skin friction coefficient or fractional drag coefficient on the surface of the moving wall is

$$
C_{f}=\frac{\left.2 \tau_{x y}\right|_{y=0}}{\rho u_{w}^{2}},
$$

Now using Equations (1)-(4) and (11) the Equation (14) can be written in dimensionless variables as

$$
C_{f}=2\left\{f^{\prime}(0, \xi)+\alpha \frac{\partial}{\partial \xi} f^{\prime}(0, \xi)+\beta \frac{\partial^{2} f^{\prime}(0, \xi)}{\partial \xi^{2}}+\frac{1}{3} \zeta\left(f^{\prime}(0, \xi)\right)^{3}\right\},
$$

\section{Analytic Solution with Convergence Check}

The boundary conditions (13) leads us to take base functions for the velocity $f(\eta, \xi)$ as

$$
\left\{\eta^{i} \xi^{j} \mathrm{e}^{-n \eta} / n \geq 0, i \geq 0, j \geq 0\right\}
$$

The velocity $f(\eta, \xi)$ can be expressed in terms of base functions as

$$
f(\eta, \xi)=\sum_{n=0}^{\infty} \sum_{i=0}^{\infty} \sum_{j=0}^{\infty} \Omega_{n, i}^{j} \eta^{i} \xi^{j} \mathrm{e}^{-n \eta},
$$

To start with the homotopy analysis method, due to the boundary conditions (13) it is reasonable to choose the initial guess approximation

$$
f_{0}(\eta, \xi)=(1-\xi \eta) \mathrm{e}^{-\eta}
$$

and the auxiliary linear operator

$$
\mathbf{L}(f)=\frac{\partial^{2} f(\eta, \xi)}{\partial \eta^{2}}-f(\eta, \xi)
$$

with the property:

$$
\mathbf{L}\left[C_{1} \mathrm{e}^{-\eta}+C_{2} \mathrm{e}^{\eta}\right]=0
$$

where $C_{1}$ and $C_{2}$ are arbitrary constants. Following the HAM and trying higher iterations with the unique and proper assignment of the results converge to the exact solution:

$$
f(\eta, \xi) \approx f_{0}(\eta, \xi)+f_{1}(\eta, \xi)+f_{2}(\eta, \xi)+\cdots+f_{m}(\eta, \xi),
$$

At $\in=0, \quad N=0.1, \alpha=0.1, \quad \beta=0.1, \zeta=0.1, \hbar=-0.5$, using the symbolic computation software such as MATLAB, MAPLE, MATHEMATICA to successively obtain

$$
\begin{aligned}
f_{1}(\eta, \xi)= & -\frac{1}{160} \mathrm{e}^{-3 \eta}+\frac{\mathrm{e}^{-\eta}}{160}+\frac{31 \eta \mathrm{e}^{-\eta}}{80}+\frac{9 \eta^{2} \mathrm{e}^{-\eta}}{80}-\frac{7}{640} \mathrm{e}^{-3 \eta} \xi+\frac{7}{640} \mathrm{e}^{-\eta} \xi+\frac{3}{160} \mathrm{e}^{-3 \eta} \eta \xi+\frac{31}{80} \mathrm{e}^{-\eta} \eta \xi \\
& -\frac{9}{80} \mathrm{e}^{-\eta} \eta^{2} \xi-\frac{13}{1280} \mathrm{e}^{-3 \eta} \xi^{2}+\frac{13}{1280} \mathrm{e}^{-\eta} \xi^{2}+\frac{7}{320} \mathrm{e}^{-3 \eta} \eta \xi^{2}-\frac{3}{160} \mathrm{e}^{-3 \eta} \eta^{2} \xi^{2}-\frac{11}{5120} \mathrm{e}^{-3 \eta} \xi^{3}, \\
& +\frac{11}{5120} \mathrm{e}^{-\eta} \xi^{3}+\frac{13}{1280} \mathrm{e}^{-3 \eta} \eta \xi^{3}-\frac{7}{640} \mathrm{e}^{-3 \eta} \eta^{2} \xi^{3}+\frac{1}{160} \mathrm{e}^{-3 \eta} \eta^{3} \xi^{3}+\mathrm{e}^{-\eta}(1-\eta \xi)
\end{aligned}
$$

similarly $f_{2}(\eta, \xi), f_{3}(\eta, \xi), f_{4}(\eta, \xi)$ and so on are calculated. The obtained values of $f_{0}(\eta, \xi), f_{1}(\eta, \xi)$, 
$f_{2}(\eta, \xi), \ldots$, leads us to write

$$
f_{m}(\eta, \xi)=\sum_{n=0}^{2 m+12 m+2-n 2 m+1} \sum_{i=0}^{i, j} \sum_{j=0}^{i, j} \Omega^{i} \xi^{j} \mathrm{e}^{-n \eta} .
$$

By avoiding the detailed recurrence relations which we calculated but not presenting here due to space, the total complex analytic solution in compact form is

$$
f(\eta, \xi)=\sum_{m=0}^{\infty} f_{m}(\eta, \xi)=\lim _{L \rightarrow \infty}\left(\sum_{m=0}^{L} \Omega_{m, 0}^{0,0}+\sum_{n=1}^{L+1} \mathrm{e}^{-n \eta}\left(\sum_{m=n-1}^{L} \sum_{i=0}^{2 m+2-n} \sum_{j=0}^{m} \Omega_{m, n}^{i, j} \eta^{i} \xi^{j}\right)\right)
$$

where from initial guess in Equation (18) we obtain

$$
\Omega_{0,1}^{0,0}=1, \Omega_{0,1}^{1,1}=-1 \text { and all } \Omega_{0, n}^{i, j}=0 \text {, for }(i=0,1,2),(j, n=0,1) .
$$

Clearly Equation (24) contains the auxiliary parameter $\hbar$, which gives the convergence region and rate of approximation for the homotopy analysis method. For this purpose, the $\hbar$-curve is plotted for the dimensionless velocity $f(\eta, \xi)$ for the sudden jerked motion of the plane wall at $\in=0$. It is obvious from Figure 1 that the range for the admissible value for $\hbar$ is $-1<\hbar<0$. Our calculations depict that the series of the dimensionless velocity in Equation (24) converges in the whole region of $\eta$ and $\xi$ for $\hbar=-0.5$.

\section{Graphs, Table and Discussion}

In order to discuss the effects of various parameters on the flow field the graphs are drawn for the variation of the dimensionless velocity $f(\eta, \xi)$ with distance from the surface $\eta$. These graphs are plotted for different values of the Hartmann number $N$, Hall parameter $\in$, homotopy parameter $\hbar$, second-grade material parameter $\alpha$, third-grade material parameters $\beta$ and $\zeta$. Figures 2 to 7 are plotted in absence of Hall currents.

Figure 2 indicates the variation of the velocity $f(\eta, \xi)$ with time $\xi$ at different distances from the wall. From Figure 2 it is observed that the velocity decays with the increase of the distance from the wall and also the fluid near the wall moves together with the wall with the same velocity as of the wall. Figure 3 shows that for fixed values of $\hbar, N, \alpha, \beta$ and $\zeta$ after the sudden jerked of the wall with increase in dimensionless time $\zeta$ the velocity $f(\eta, \xi)$ decreases at all points and decays with increase of time, boundary layer thickness decreases, which results in shear thinning effects, which are used in recognition of the fluid. Clearly the viscosity induces in the fluid with the passage of time and after some time the steady state is achieved which proves the uniform validity of the solution for all time in the whole spatial region $0 \leq \eta<\infty$. Figure 4 illustrates that with increase in Hartmann number $N$ the velocity $f(\eta, \xi)$ decreases and boundary layer thickness also decreases at all points. When magnetic field is applied transverse to the fluid velocity then it gives rise to a drag-like or resistive force which slow down or suppress the motion of the fluid on the wall. This leads to a reduction in the velocity of the fluid as seen in Figure 4. Figure 5 demonstrate the velocity changes with distance from the wall

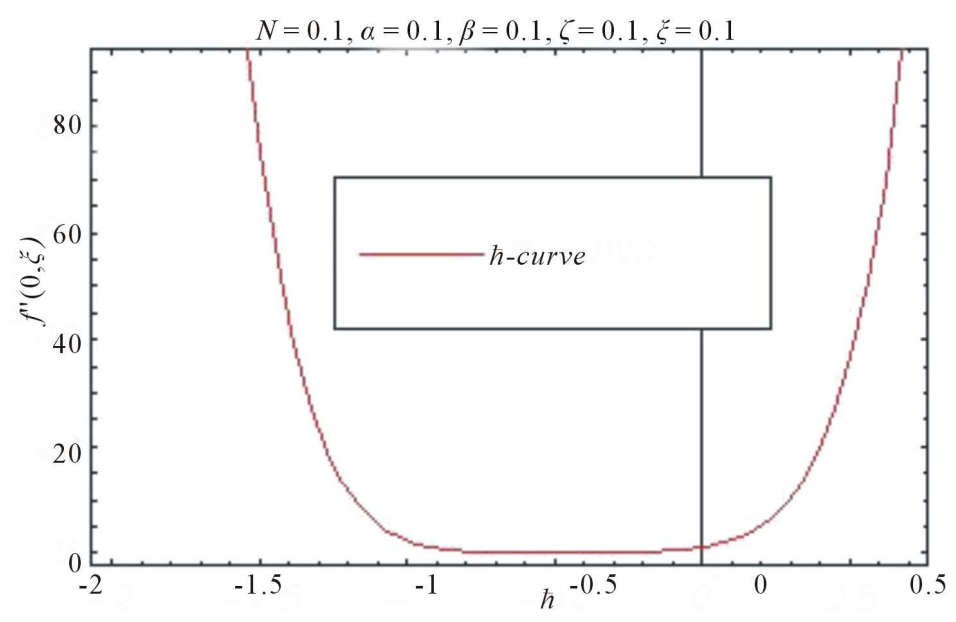

Figure 1. $\hbar$-curve for $f(\eta, \xi)$. 


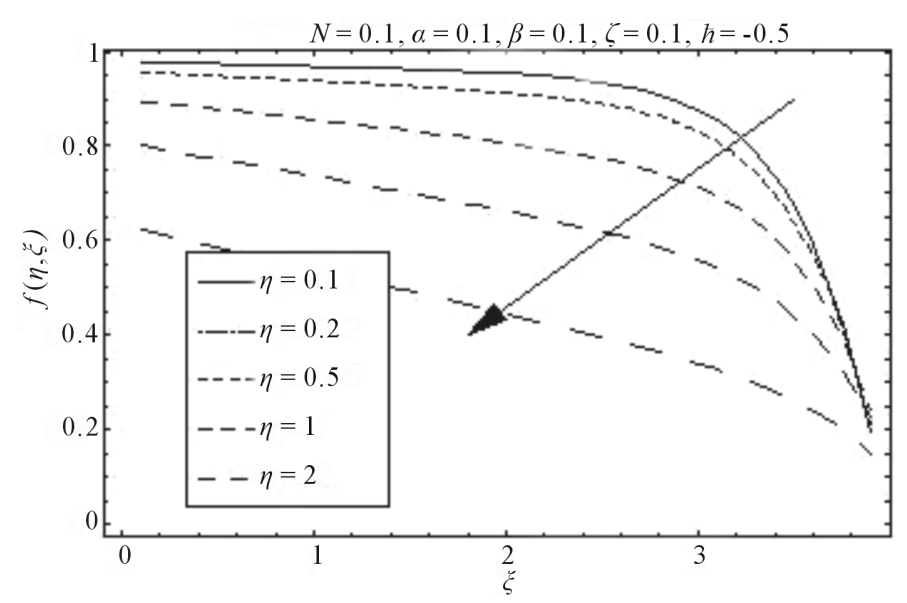

Figure 2. Influence of $\eta$ on $f(\eta, \xi)$.

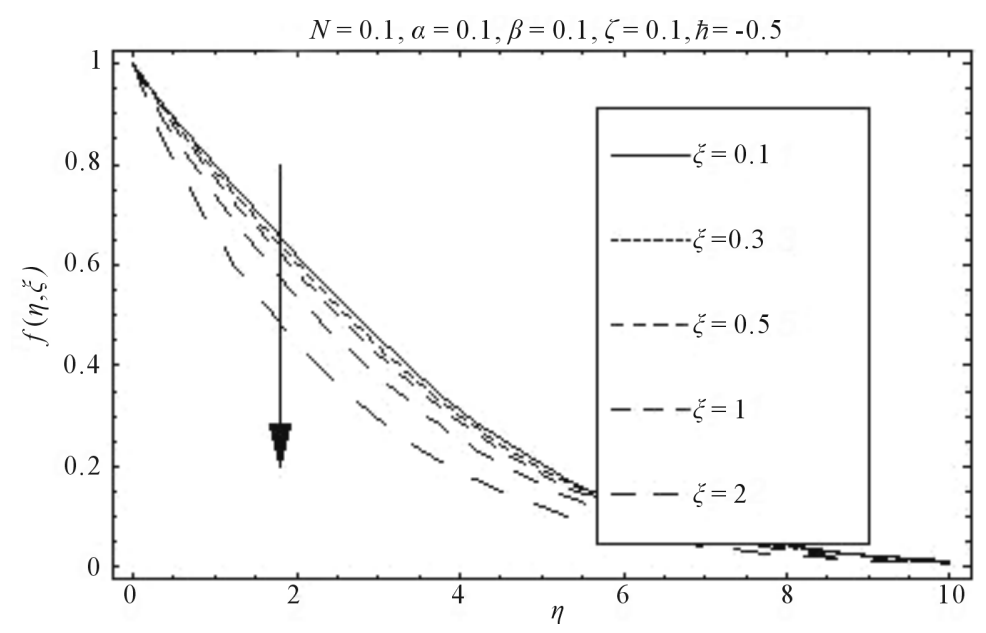

Figure 3. Influence of $\xi$ on $f(\eta, \xi)$.

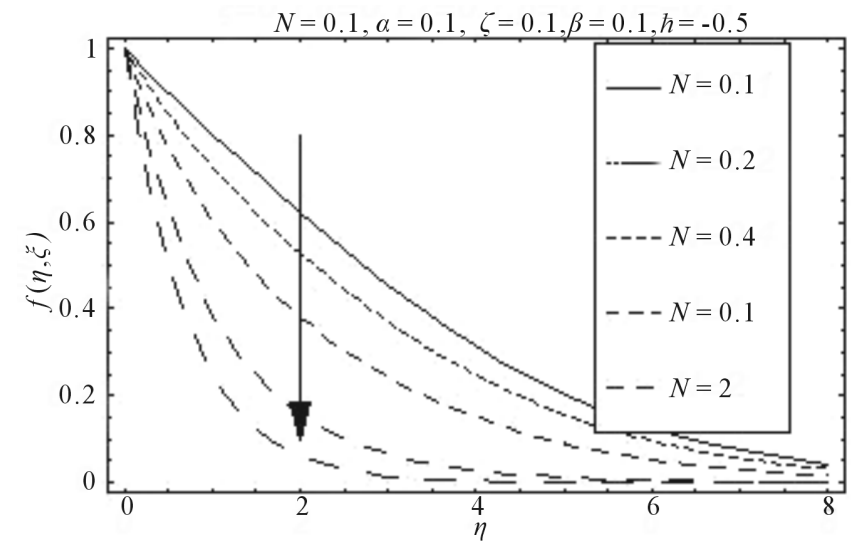

Figure 4. Influence of $N$ on $f(\eta, \xi)$.

at given points $(\eta=1)$ and $(\eta=4)$. It is clearly seen that the smaller the $\alpha$, the more rapidly the velocity changes very close to the wall and the more like a solid the third grade fluid behaves. After $\eta=4$ velocity decreases, indicating larger apparent viscosity for third-grade fluid. Figure 6 displays that for fixed values of $\hbar$, 


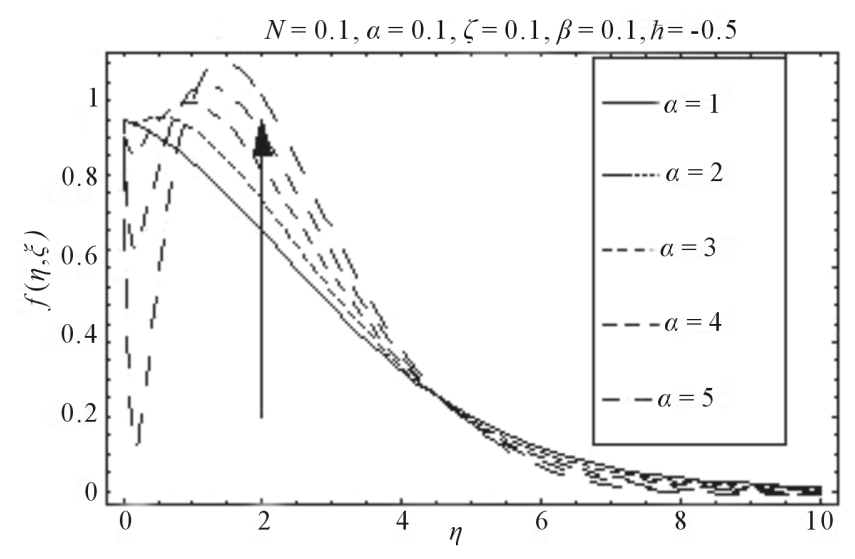

Figure 5. Influence of $\alpha$ on $f(\eta, \xi)$.

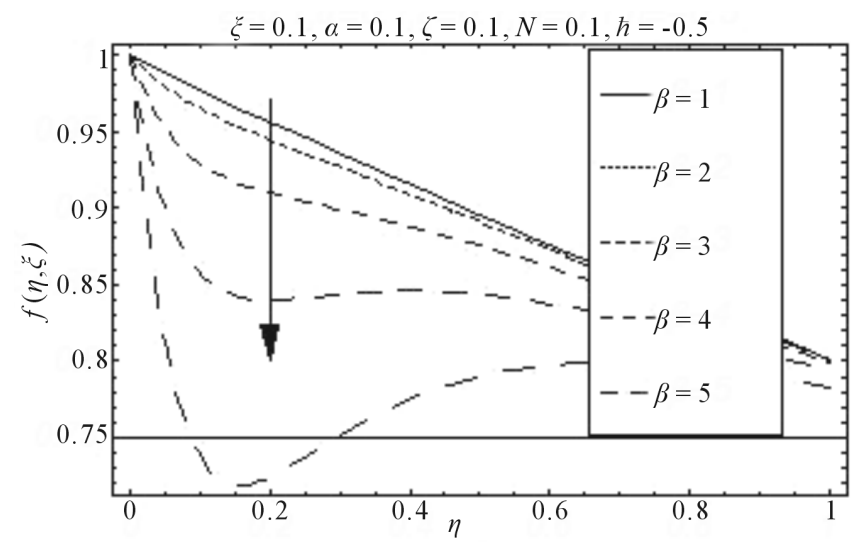

Figure 6. Influence of $\beta$ on $f(\eta, \xi)$.

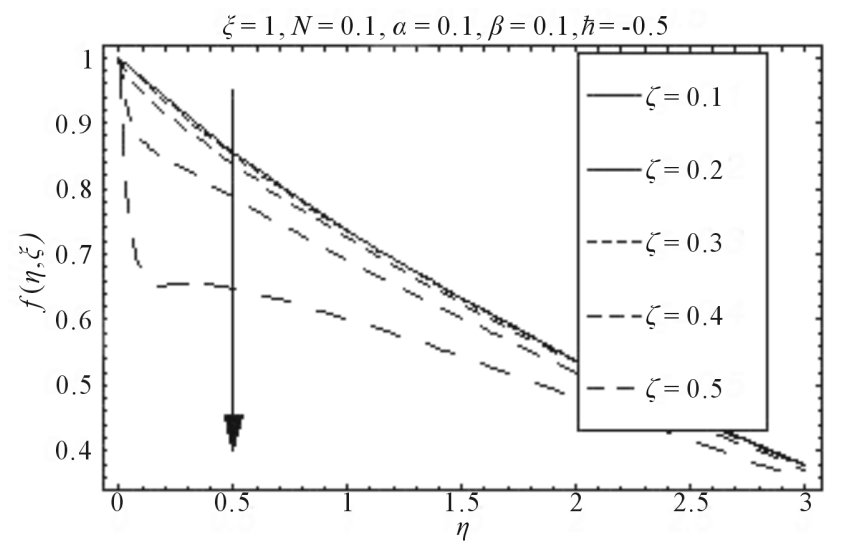

Figure 7. Influence of $\zeta$ on $f(\eta, \xi)$.

$\xi, N, \alpha$ and $\zeta$ with increase in third-grade material parameter $\beta$ the velocity $f(\eta, \xi)$ and boundary layer thickness decreases at all points. Figure 7 illustrates that for fixed values of $\hbar, \xi, N, \beta$ and $\alpha$ with increase in third-grade material parameter $\zeta$ the velocity $f(\eta, \xi)$ and boundary layer thickness decreases. Inclusion of Hall current $\in$ makes the velocity field complex, so we plot real and imaginary parts of the velocity to see the effects of Hall current on the flow field. Figure 8 describes that for fixed values of $\hbar, N, \xi, \alpha$, $\beta$ and $\zeta$ with increase in Hall parameter $\in$ real part of the velocity decreases in magnitude, boundary layer 
thickness increases, which results in shear thickening effects. Figure 9 shows that for fixed values of $\hbar, N$, $\xi, \alpha, \beta$ and $\zeta$ with increase in Hall parameter $\in$ imaginary part of the velocity decreases in magnitude and boundary layer thickness increases.

Here the table is prepared for the variation of the absolute values of the skin friction coefficient $C_{f}$ given by Equation (15) for sudden jerked motion of the wall. The table shows the impact of the Hartmann number $N$ and Hall current $\in$ with time $\xi$ on $C_{f}$. It is observed from table that for fixed values of $\zeta, \hbar, \alpha, \beta$ and $\in$ with increase in Hartmann number $N$ absolute value of the skin friction coefficient $C_{f}$ increases for all values of the time $\xi$. Also for fixed values of $N, \hbar, \alpha, \beta$ and $\zeta$ with increase in Hall current $\in$ absolute value of the skin friction coefficient decreases for all values of the time $\xi$. For fixed values of $N, \hbar, \alpha$, $\beta, \zeta$ and $\in$ with increase in dimensionless time $\xi$ the absolute value of the skin friction coefficient increases at all points. When magnetic field is applied normal to the fluid velocity then it gives rise to a drag-like or resistive force which slow down or suppress the motion of the fluid on the moving surface. This leads to a reduction in the velocity of the fluid and flow rates. With the increase in the strength of the magnetic field the motion of the particulate suspension on the surface reduces due to which shear stress at the wall reduces with increase in Hall current $\in$, as observed in the Table 1.

\section{Conclusion}

The Stokes' first problem of an unsteady MHD third grade fluid in a non-porous half space is discussed by tak-

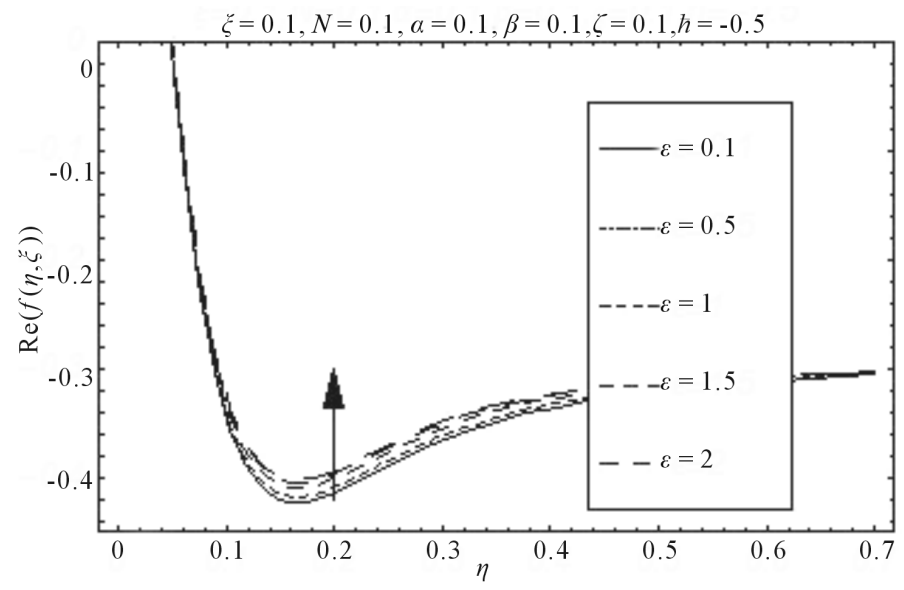

Figure 8. Influence of $\in$ on $\operatorname{Re}(f(\eta, \xi))$.

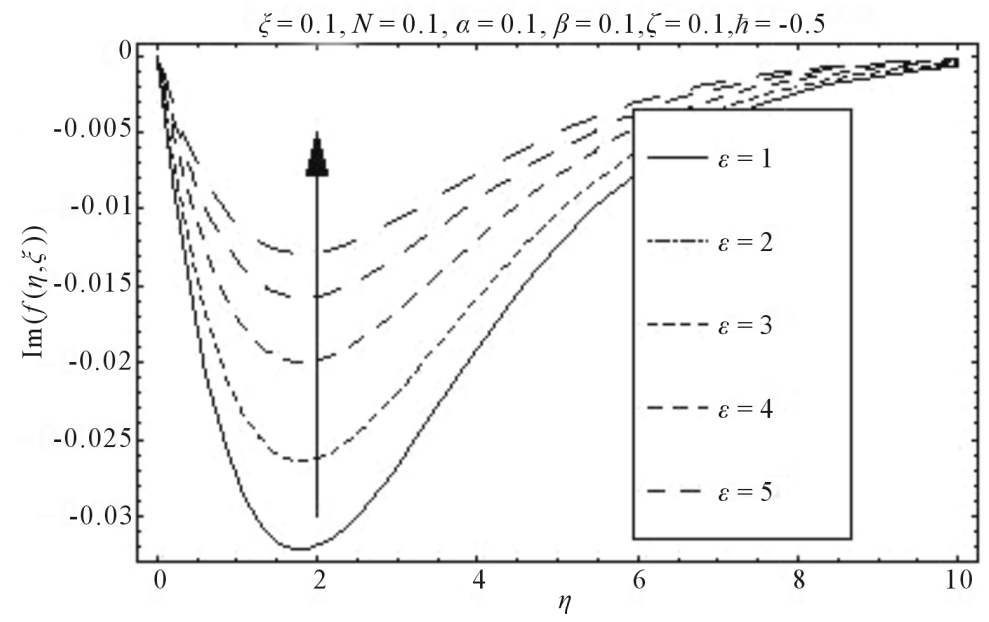

Figure 9. Influence of $\in$ on $\operatorname{Im}(f(\eta, \xi))$. 
Table 1. Absolute values of the skin friction coefficient $C_{f}$ with $\alpha=0.1, \beta=0.1, \zeta=0.1, \hbar=-0.5$.

\begin{tabular}{ccccccc}
\hline$\xi$ & $\epsilon=0.1$ & $\epsilon=0.1$ & $\epsilon=0.1$ & $N=0.1$ & $N=0.1$ & $N=0.1$ \\
\hline 0.2 & $N=0.1$ & $N=0.3$ & $N=0.5$ & $\epsilon=0.2$ & $\epsilon=0.3$ & $\epsilon=0.5$ \\
0.4 & 1.07115 & 1.24636 & 1.41974 & 1.06873 & 1.06496 & 1.05487 \\
0.6 & 1.23092 & 1.39239 & 1.55308 & 1.22867 & 1.22517 & 1.21582 \\
0.8 & 1.39330 & 1.54134 & 1.68946 & 1.39122 & 1.38800 & 1.37940 \\
1 & 1.55974 & 1.69467 & 1.83052 & 1.55784 & 1.55490 & 1.54705 \\
\hline
\end{tabular}

ing Hall currents into account. Analytical solution for the non-linear problem is given and convergence of the solution is appropriately discussed. The non-linear effect on the velocity is shown and discussed. It reveals that plots for shear thickening/shear thinning behavior of a fluid are dependent upon the rheological properties of the fluid. The numerical results for Hall parameter $\in$, Hartmann number $N$, second-grade parameter $\alpha$, thirdgrade material parameters $\beta, \zeta$ and dimensionless time $\zeta$ reveal that Hall parameter, Hartmann number and dimensionless time have significant influence on the local skin-friction coefficient on the surface of the impulsively moving wall.

\section{References}

[1] Stokes, G.G. (1851) On the Effect of the Internal Friction of Fluids on the Motion of Pendulums. Transactions of the Cambridge Philosophical Society, Part II, 9, 8-106.

[2] Tanner, R.I. (1962) Note on the Rayleigh Problem for a Visco-Elastic Fluid. Zeitschrift für Angewandte Mathematik und Physik, 13, 573-580. http://dx.doi.org/10.1007/BF01595580

[3] Soundalgekar, V.M. (1974) Stokes Problem for Elastico-Viscous Fluid. Rheologica Acta, 13, 177-179. http://dx.doi.org/10.1007/BF01520872

[4] Teipel, I. (1981) The Impulsive Motion of a Flat Plate in a Viscoelastic Fluid. Acta Mechanica, 39, 277-279. http://dx.doi.org/10.1007/BF01170349

[5] Puri, P. (1984) Impulsive Motion of a Flat Plate in a Rivlin-Ericksen Fluid. Rheologica Acta, 23, 451-453. http://dx.doi.org/10.1007/BF01329198

[6] Erdogan, M.E. (1995) Plane Surface Suddenly Set in Motion in a Non-Newtonian Fluid. Acta Mechanica, 108, 179187. http://dx.doi.org/10.1007/BF01177337

[7] Zeng, Y. and Weinbaum, S. (1995) Stokes Problems for Moving Half-Planes. Journal of Fluid Mechanics, 287, 59-74. http://dx.doi.org/10.1017/S0022112095000851

[8] Tan, W.C. and Xu, M.Y. (2002) The Impulsive Motion of Flat Plate in a Generalized Second Grade Fluid. Mechanics Research Communications, 29, 3-9. http://dx.doi.org/10.1016/S0093-6413(02)00223-9

[9] Tan, W.C. and Xu, M.Y. (2002) Plane Surface Suddenly Set in Motion in a Viscoelastic Fluid with Fractional Maxwell Model. Acta Mechanica Sinica, 18, 342-349. http://dx.doi.org/10.1007/BF02487786

[10] Erdogan, M.E. (2002) On the Unsteady Unidirectional Flows Generated by Impulsive Motion of a Boundary or Sudden Application of a Pressure Gradient. International Journal of Non-Linear Mechanics, 37, 1091-1106. http://dx.doi.org/10.1016/S0020-7462(01)00035-X

[11] Fetecau, C. and Fetecau, C. (2003) The First Problem of Stokes for an Oldroyd-B Fluid. International Journal of NonLinear Mechanics, 38, 1539-1544. http://dx.doi.org/10.1016/S0020-7462(02)00117-8

[12] Tan, W.C. and Masuoka, T. (2005) Stokes' First Problem for an Oldroyd-B Fluid in a Porous Half Space. Physics of Fluids, 17, 023101. http://dx.doi.org/10.1063/1.1850409

[13] Tan, W.C. and Masuoka, T. (2005) Stokes' First Problem for a Second Grade Fluid in a Porous Half-Space with Heated Boundary. International Journal of Non-Linear Mechanics, 40, 515-522. http://dx.doi.org/10.1016/j.ijnonlinmec.2004.07.016

[14] Zierep, J. and Fetecau, C. (2007) Energetic Balance for the Rayleigh-Stokes Problem of a Second Grade Fluid. International Journal of Engineering Science, 45, 155-162. http://dx.doi.org/10.1016/j.ijengsci.2006.09.001

[15] Zierep, J., Bohning, R. and Fetecau, C. (2007) Rayleigh-Stokes Problem for Non-Newtonian Medium with Memory. 
Zeitschrift für Angewandte Mathematik und Mechanik, 87, 462-467. http://dx.doi.org/10.1002/zamm.200710328

[16] Vieru, D., Nazar, M., Fetecau, C. and Fetecau, C. (2008) New Exact Solutions Corresponding to the First Problem of Stokes for Oldroyd-B Fluids. Computers \& Mathematics with Applications, 55, 1644-1652. http://dx.doi.org/10.1016/j.camwa.2007.04.040

[17] Shahzad, F., Hayat, T. and Ayub, M. (2008) Stokes’ First Problem for the Rotating Flow of a Third Grade Fluid. Nonlinear Analysis: Real World Applications, 9, 1794-1799. http://dx.doi.org/10.1016/j.nonrwa.2007.05.008

[18] Hayat, T., Shahzad, F., Ayub, M. and Asghar, S. (2008) Stokes' First Problem for a Third Grade Fluid in a Porous Half Space. Communications in Nonlinear Science and Numerical Simulation, 13, 1801-1807. http://dx.doi.org/10.1016/j.cnsns.2007.04.015

[19] Fakhari, K., Zainal, A.A. and Kara, A.H. (2011) A Note on the Interplay between Symmetries, Reduction and Conservation Laws of Stokes’ First Problem for Third-Grade Rotating Fluids. Pramana Journal of Physics, 77, 439-445. http://dx.doi.org/10.1007/s12043-011-0164-6

[20] Sajid, M., Ali, N., Javed T. and Abbas, Z. (2010) Stokes' First Problem for a MHD Third Grade Fluid in a Porous Half Space. Journal of Porous Media, 1, 279-284.

[21] Sato, H. (1961) The Hall Effects in the Viscous Flow of Ionized Gas between Parallel Plates under Transverse Magnetic Field. Journal of the Physical Society of Japan, 16, 1427-1433. http://dx.doi.org/10.1143/JPSJ.16.1427

[22] Cramer, K. and Pai, S. (1973) Magnetofluid Dynamics for Engineers and Applied Physicists. McGraw-Hill, New York.

[23] Ayub, M., Zaman, H. and Ahmad, M. (2010) Series Solution of Hydromagnetic Flow and Heat Transfer with Hall Effect in a Second Grade Fluid over a Stretching Sheet. Central European Journal of Physics, 8, 135-149. http://dx.doi.org/10.2478/s11534-009-0110-0

[24] Ahmad, M., Zaman, H. and Rehman, N. (2010) Effects of Hall Current on Unsteady MHD Flows of a Second Grade Fluid. Central European Journal of Physics, 8, 422-431. http://dx.doi.org/10.2478/s11534-009-0083-z

[25] Zaman, H. (2013) Hall Effects on the Unsteady Incompressible MHD Fluid Flow with Slip Conditions and Porous Walls. Applied Mathematics and Physics, 1, 31-38.

[26] Hayat, T., Zaman, H. and Ayub, M. (2010) Analytic Solution of Hydromagnetic Flow with Hall Effect over a Surface Stretching with a Power Law Velocity. Numerical Methods for Partial Differential Equations, 27, 937-959.

[27] Hayat, T., Naz, R. and Asghar, S. (2004) Hall Effects on Unsteady Duct Flow of a Non-Newtonian Fluid in a Porous Medium. Applied Mathematics and Computation, 157, 103-114. http://dx.doi.org/10.1002/num.20562

[28] Hayat, T. and Nawaz, M. (2011) Hall and Ion-Slip Effects on Three-Dimensional Flow of a Second Grade Fluid. International Journal for Numerical Methods in Fluids, 66, 183-193. http://dx.doi.org/10.1016/j.amc.2003.08.069

[29] Pop I. and Soundalgekar, V.M. (1974) Effects of Hall Current on Hydromagnetic Flow near a Porous Plate. Acta Mechanica, 20, 315-318. http://dx.doi.org/10.1007/BF01175933

[30] Gupta, A.S. (1975) Hydromagnetic Flow Past a Porous Flate Plate with Hall Effects. Acta Mechanica, 22, $281-287$. http://dx.doi.org/10.1007/BF01170681

[31] Debnath, L., Ray, S.C. and Chatterjee, A.K. (1979) Effects of Hall Current on Unsteady Hydromagnetic Flow Past a Porous Plate in a Rotating Fluid System. Zeitschrift für Angewandte Mathematik und Mechanik, 59, 469-471. http://dx.doi.org/10.1002/zamm.19790590910

[32] Katagiri, M. (1969) The Effect of Hall Currents on the Magnetohydrodynamic Boundary Layer Flow Past a Semi-Infinite Flate Plate. Journal of the Physical Society of Japan, 27, 1051-1059. http://dx.doi.org/10.1143/JPSJ.27.1051

[33] Abo-Eldahab, E.M. and Elbarbary, M.E. (2001) Hall Current Effect on Magnetohydrodynamic Free Convection Flow Past a Semi-Infinite Vertical Plate with Mass Transfer. International Journal of Engineering Science, 39, 1641-1652. http://dx.doi.org/10.1016/S0020-7225(01)00020-9

[34] Abo-Eldahab, E.M. and Salem, A.M. (2004) Hall Effects on MHD Free Convection Flow of a Non-Newtonian Power Law Fluid at a Stretching Surface. International Communications in Heat and Mass Transfer, 31, 343-354. http://dx.doi.org/10.1016/j.icheatmasstransfer.2004.02.005

[35] Attia, H.A. (2006) Hall Effects on the Flow of a Dusty Bingham Fluid in a Circular Pipe. Turkish Journal of Engineering and Environmental Sciences, 30, 14-21.

[36] Attia, H.A. (1998) Hall Current Effects on the Velocity and Temperature Fields of an Unsteady Hartman Flow. Canadian Journal of Physics, 76, 739-746.

[37] Liao, S.J. (2003) Beyond Perturbation: Introduction to Homotopy Analysis Method. Chapman and Hall/CRC Press, Florida. http://dx.doi.org/10.1201/9780203491164

[38] Liao, S.J. (1992) The Proposed Homotopy Analysis Technique for the Solution of Nonlinear Problem. Ph.D. Thesis, Shanghai Jiao Tong University, Shanghai. 
[39] Liao, S.J. (2012) Homotopy Analysis Method in Nonlinear Differential Equations. Springer-Verlag, Berlin. http://dx.doi.org/10.1007/978-3-642-25132-0

[40] Liao, S.J. (2013) Advances in the Homotopy Analysis Method. World Scientific Publishing Company, Singapore.

[41] Liao, S.J. (2009) Notes on the Homotopy Analysis Method: Some Definitions and Theorems. Communications in Nonlinear Science and Numerical Simulation, 14, 983-997.

[42] Liao, S.J. (2006) An Analytic Solution of Unsteady Boundary-Layer Flows Caused by an Impulsively Stretching Plate. Communications in Nonlinear Science and Numerical Simulation, 11, 326-339.

[43] Ayub, M., Zaman, H., Sajid, M. and Hayat, T. (2008) Analytical Solution of Stagnation-Point Flow of a Viscoelastic Fluid towards a Stretching Surface. Communications in Nonlinear Science and Numerical Simulation, 13, 1822-1835. http://dx.doi.org/10.1016/j.cnsns.2007.04.021

[44] Zaman, H. and Ayub, M. (2010) Series Solution of Unsteady Free Convection Flow with Mass Transfer along an Accelerated Vertical Porous Plate with Suction. Central European Journal of Physics, 8, 931-939. http://dx.doi.org/10.2478/s11534-010-0007-y

[45] Zaman, H., Hayat, T., Ayub, M. and Gorla, R.S.R. (2011) Series Solution for Heat Transfer from a Continuous Surface in a Parallel Free Stream of Viscoelastic Fluid. Numerical Methods for Partial Differential Equations, 27, 1511-1524. http://dx.doi.org/10.1002/num.20593

[46] Rivlin, R.S. and Ericksen, J.L. (1955) Stress Deformation Relations for Isotropic Materials. Journal of Rational Mechanics and Analysis, 4, 323-425.

[47] Sutton, G.W. and Sherman, A. (1965) Engineering Magnetohydrodynamics. McGraw-Hill, New York. 